\title{
Numerical Investigation of Flow Transition Behavior in Cold Flow Dual Bell Rocket Nozzles
}

\author{
Dirk Schneider* and Chloé Génin ${ }^{\dagger}$ \\ German Aerospace Center (DLR), Lampoldshausen, D-74239, Germany
}

\begin{abstract}
The dual bell nozzle is an altitude-adaptive nozzle concept. It combines the advantages of a nozzle with small area ratio under sea-level conditions and a large area ratio nozzle under high altitude conditions. RANS and URANS simulations were conducted at the German Aerospace Center (DLR) in Lampoldshausen, to investigate the transition from one mode to the other of a dual bell nozzle model with positive pressure gradient ( $\mathrm{pp}$ ) extension. A cold flow test campaign conducted at DLR's cold flow test facility P6.2 provided validation data for the numerical approach. The present study investigates the influence of different turbulence models and feeding pressure gradients on the dual bell flow transition behavior. Best results were achieved for the Spalart-Allmaras and RSM turbulence model. A clear impact of the feeding pressure ramp on the dual bell transition pressure ratio and the flow separation position velocity was shown. The transition NPR was predicted with an accuracy of one percent. For the hysteresis between transition and retransition nozzle pressure ratio, an accuracy of approximately ten percent was reached. The calculated values of the experimental and numerical transition duration were on the same order of magnitude.
\end{abstract}

\section{Nomenclature}

$\begin{array}{ll}A & \text { Area } \\ H & \text { Hysteresis gap } \\ L & \text { Length } \\ R & \text { Radius } \\ y^{+} & \text {Dimensionless wall spacing }\end{array}$

Subscripts

$\mathrm{b}$

e

i

retr

th

tr

t

Conventions

AUSM

cp

MUSCL

NPR

pp

RANS

SST

TIC

\author{
Base nozzle \\ Nozzle extension \\ Inflection \\ Retransition \\ Throat \\ Transition \\ Total
}

\footnotetext{
*PhD Student, Institute of Space Propulsion, Langer Grund, Lampoldshausen. dirk.schneider@dlr.de. Member AIAA. ${ }^{\dagger}$ Research Scientist, Institute of Space Propulsion, Langer Grund, Lampoldshausen. Member AIAA.
} 


\section{Introduction}

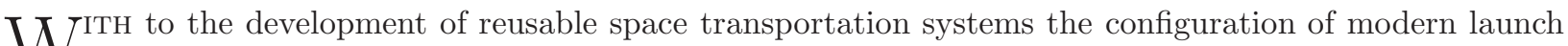
$W$ vehicles has changed from serial to parallel staging. One example of a parallel staged launch vehicle is the European heavy launcher Ariane 5. Here the main stage engine is ignited prior to the booster stage, to ensure correct operation of the engine before lift-off. Therefore, the main stage engine has to operate over a wide range of altitudes, from sea-level up to near-vacuum conditions. To avoid asymmetrical flow separation of the highly over-expanded nozzle flow at low altitude, the area ratio of conventional nozzles is limited. Thus, side loads induced by asymmetrical flow separation only occur during start-up of the main stage engine. The limitation of the nozzle area ratio leads to a reduced engine performance at high altitudes.

The dual bell nozzle ${ }^{1,2}$ combines the advantage of a nozzle with small area ratio under sea-level conditions and a high area ratio nozzle under altitude conditions. It is composed of a base nozzle and a nozzle extension linked by an abrupt change in wall angle. Under high ambient pressure this contour inflection forces the flow to a controlled and symmetrical flow separation. The nozzle is in sea-level mode. During ascent of the launch vehicle the ambient pressure decreases. At a certain altitude the nozzle pressure ratio (ratio of total pressure over ambient pressure) NPR reaches a value at which transition to altitude mode takes place. This means the

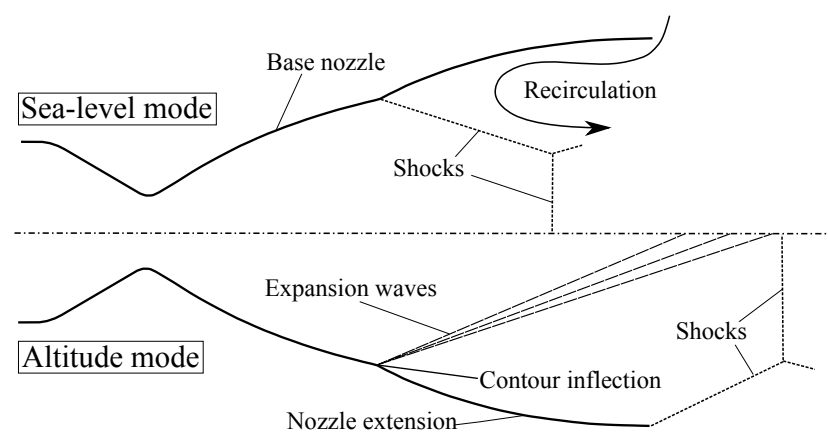

Figure 1. Principle of a dual bell nozzle. separation position of the highly over-expanded nozzle flow suddenly moves to the exit plane of the dual bell nozzle extension. Due to the increased expansion of the combustion gases, the performance of the engine is increased ${ }^{3}$ compared to an engine using a conventional nozzle with limited area ratio. The principle of a dual bell nozzle is illustrated in Figure 1. Sea-level mode (top) and altitude mode (bottom) are both well known stable operation conditions and are of lesser interest in scientific research. The main focus is on the transition from one mode to the other. Between transition and retransition NPR, a hysteresis effect exists. This hysteresis effect prevents the dual bell nozzle from changing modes just by small fluctuations in combustion chamber or ambient pressure.

\section{Experimental Setup}

Validation data for the numerical approach were provided by cold flow test campaigns performed at DLR's test facility P6.2 in Lampoldshausen. At the test bench dry gaseous nitrogen is used as feeding fluid.

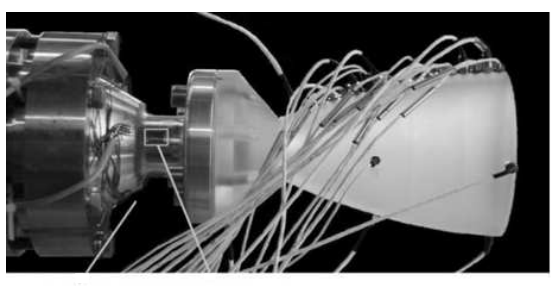

Sea-level mode

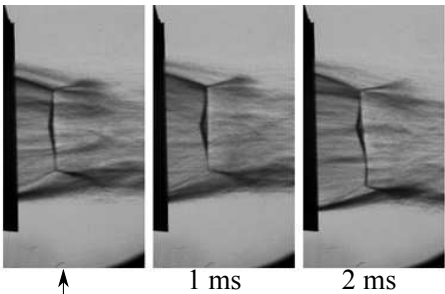

$1 \mathrm{~ms}$

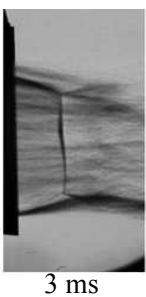

$3 \mathrm{~ms}$
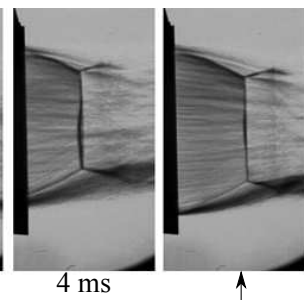

Altitude mode

Figure 2. Experimental setup at test facility P6.2 and Schlieren imaging of the transition from sea-level to altitude mode.

The experiments were conducted on the horizontal test rig under sea-level conditions. The feeding pressure 
of the nitrogen was progressively varied to reach different values of NPR. Several test profiles were applied, with feeding pressure gradients between 0.5 and $2.5 \mathrm{bar} / \mathrm{s}$. A maximum NPR of 55 was reached during test. The picture on the left side of Figure 2 illustrates the nozzle model mounted on the horizontal test rig.

Dynamic pressure transducers were placed along the nozzle wall from nozzle throat to nozzle exit plane, to provide wall pressure distributions and track the separation position of the over-expanded nozzle flow. For greater spatial resolution, additional sensors were placed in circumferential direction in the contour inflection region. Based on the experimental wall pressure data, the transition and retransition NPR, the hysteresis gap, and the transition duration were determined. The shock pattern of the nozzle plume was visualized using Schlieren imaging. Figure 2 illustrates Schlieren images taken during transition from sea-level to altitude mode. The location and angles of the shock pattern were read out of the Schlieren images and used for validation of the numerical results. Detailed information about the experimental setup are given by Génin and Stark. ${ }^{4}$

For heat conduction and manufacturing reasons, the nozzle model consisted of Polymethylmethacrylat (PMMA). To avoid interaction of an internal shock with the shock pattern in the nozzle plume, the base nozzle was designed as a truncated ideal contour (TIC). For the present study, a nozzle extension with a positive wall pressure gradient (pp) was chosen. This configuration was proved to ensure a fast transition from sea-level to altitude mode and to have nonproblematic behavior for numerical simulations. ${ }^{5}$ Figure 3 illustrates the geometrical parameters of the investigated dual bell nozzle. The

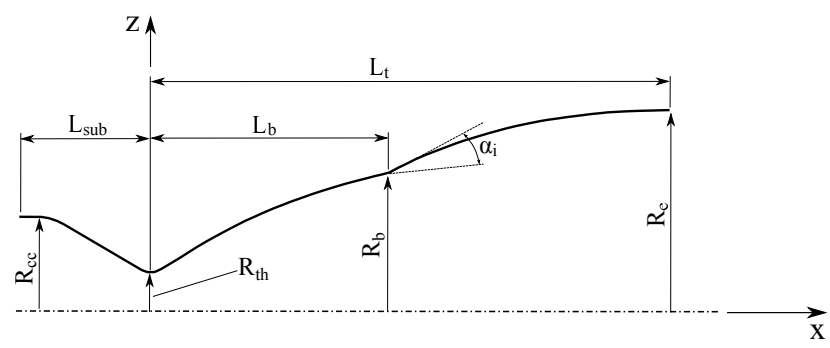

Figure 3. Geometry parameters of the dual bell nozzle model. nozzle had a throat radius of $10 \mathrm{~mm}$ and a total length of $145 \mathrm{~mm}$. The base nozzle and nozzle extension were linked by a contour inflection angle of $7.2^{\circ}$. The base nozzle had a length of $62 \mathrm{~mm}$ and an area ration of 11.3. Area ration of the nozzle extension was 25.6 with a length of $83 \mathrm{~mm}$. Table 1 gives an overview of the geometrical parameters of the investigated dual bell nozzle.

Table 1. Geometrical parameter of the investigated dual bell nozzle model

\begin{tabular}{lcc} 
Name & Symbol & Value \\
\hline Throat radius & $R_{\mathrm{th}}$ & $10 \mathrm{~mm}$ \\
Area ratio base nozzle & $\epsilon_{\mathrm{b}}=A_{\mathrm{b}} / A_{\mathrm{th}}$ & 11.3 \\
Area ratio extension & $\epsilon_{\mathrm{e}}=A_{\mathrm{e}} / A_{\mathrm{th}}$ & 25.6 \\
Base nozzle length & $L_{\mathrm{b}} / R_{\mathrm{th}}$ & 6.2 \\
Extension length & $L_{\mathrm{e}} / R_{\mathrm{th}}$ & 8.3 \\
Total nozzle length & $L_{\mathrm{t}} / R_{\mathrm{th}}$ & 14.5 \\
Inflection angle & $\alpha_{\mathrm{i}}$ & $7.2^{\circ}$
\end{tabular}

\section{Numerical Investigation}

\section{A. Numerical Method}

1. The DLR-TAU-Code

The numerical study was performed with the second order finite-volume DLR-Navier-Stokes flow solver TAU, ${ }^{6}$ which has been validated for a wide range of steady and unsteady sub-, trans- and hypersonic flows. ${ }^{7}$ For the present investigation, TAU was applied to solve the Reynolds-averaged Navier-Stokes equations on two-dimensional and axisymmetric hybrid structured/unstructured grids. Turbulence modeling was conducted using one-equation, two-equation and Reynolds-stress models. The main turbulence model for the present investigation was the Spalart-Allmaras turbulence model, ${ }^{8}$ which yielded good results for the prediction of separation positions in over-expanded nozzle flows. ${ }^{9}$ For discretization of the inviscid flux terms the 
AUSMDV ${ }^{10}$ upwind scheme was applied. A MUSCL-type gradient reconstruction was used to achieve second order spatial accuracy. Viscous terms were computed with a second order central scheme. Time integration was performed by an implicit backward Euler method with LU-SGS scheme. Time-accurate solutions were obtained using a Jameson-type dual time-stepping scheme ${ }^{11}$ with second order temporal discretization. Different physical time-step sizes were applied between $5 \cdot 10^{-7}$ and $1 \cdot 10^{-5} \mathrm{~s}$. The nitrogen was modelled as calorically perfect gas and the relationship between viscosity and temperature was defined by Sutherland's law.

\section{Numerical Computation Domain}

The present numerical study was performed on two-dimensional hybrid computational grids with approx. 500,000 grid points. The ambient conditions around the nozzle were modelled by applying a flux-based farfield boundary condition. ${ }^{12}$ To minimize the influence of the far-field boundary conditions, the dimensions of the computational domain were $30 L_{\mathrm{t}}$ in the $\mathrm{x}$-direction by $10 L_{\mathrm{t}}$ in the $\mathrm{z}$-direction. For all investigations conducted, axi-symmetric flow was assumed. At the nozzle inlet a reservoir boundary condition was applied.

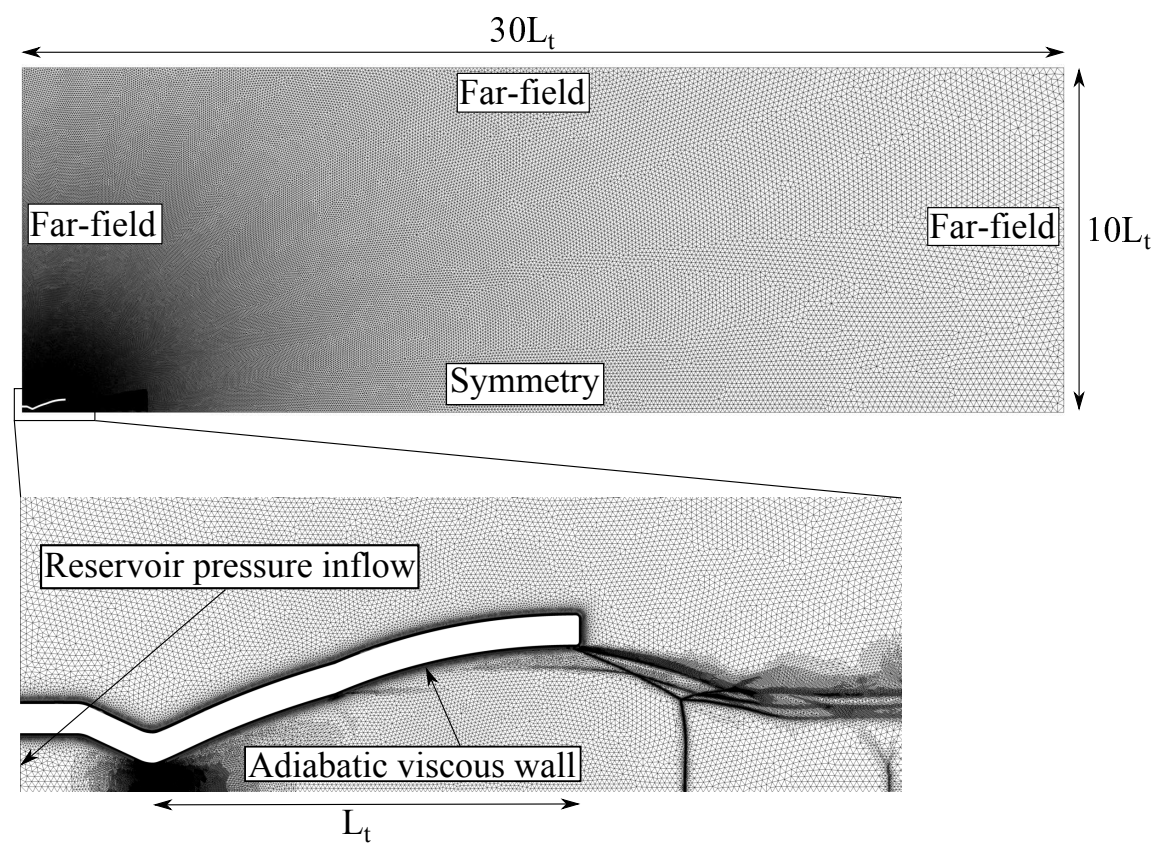

Figure 4. Numerical computation domain, boundary conditions and adapted nozzle grid.

The total feeding pressure and density were specified and the corresponding flow conditions were computed by isentropic expansion of the total conditions. For the transient computations, a linear variation of the total reservoir feeding pressure with time was performed. Total temperature of the inlet and the far-field boundary condition were set to $300 \mathrm{~K}$. At the nozzle walls, prismatic sublayers were used to resolve the viscous boundary layer. Turbulent boundary layers along adiabatic nozzle walls were assumed. To ensure a sufficient resolution of the laminar sublayer, a dimensionless wall spacing of $y^{+}=\mathrm{O}(1)$ was applied. A detailed grid study was conducted to show the grid independence of the obtained results. Furthermore, a pressure gradient indicator was used to control the fully automated grid adaptation in the vicinity of shocks and shear layers. Figure 4 illustrates the computational domain (top) and the adapted grid (bottom).

\section{B. Steady State Transition Behavior}

Steady state RANS simulations at different nozzle pressure ratios between NPR 30 and 60 were conducted, to investigate the transition behavior of the dual bell nozzle model with pp extension. Initial conditions for each NPR step were the results of the previous step. Grid adaptation was used to refine the computational grid in regions with strong pressure gradients. Therefore, five adaptation steps were applied, all of which reached full convergence after each adaptation step. For each steady state result, the separation position 
of the dual bell nozzle flow was determined. Figure 5 illustrates the flow separation positions for each of the 30 steady state simulations. Between NPR 30 and 37 a clear sea-level mode can be observed. The flow separates at the vicinity of the contour inflection point. At NPR 38 the so called sneak transition ${ }^{4}$ starts. $^{2}$ The flow separation position slowly moves inside the nozzle extension. Between NPR 42 and 43 the flow separation position moves almost to the nozzle exit plane. Transition to altitude mode takes place and the transition NPR is 43. Afterwards the dual bell nozzle is in altitude mode and the flow separates at the exit plane of the nozzle extension. Figure 6 illustrates the Mach number distribution for four different values of NPR. Between NPR 42 and 43 a clear transition to altitude mode can be observed. After transition to altitude mode, the flow separation position doesn't yet reach the very end of the nozzle. This behavior is called 'end effect' and is well known for over-expanded nozzle flows. For NPR 30 and 60, the nozzle is in clear sea-level and altitude mode, respectively.

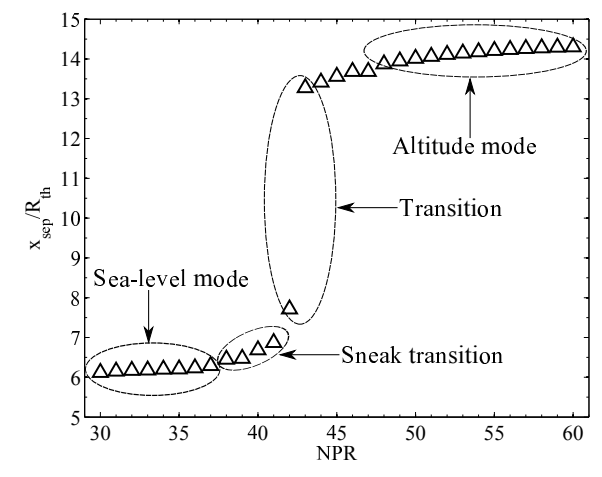

Figure 5. Separation positions in a dual bell nozzle for different values of nozzle pressure ratio.

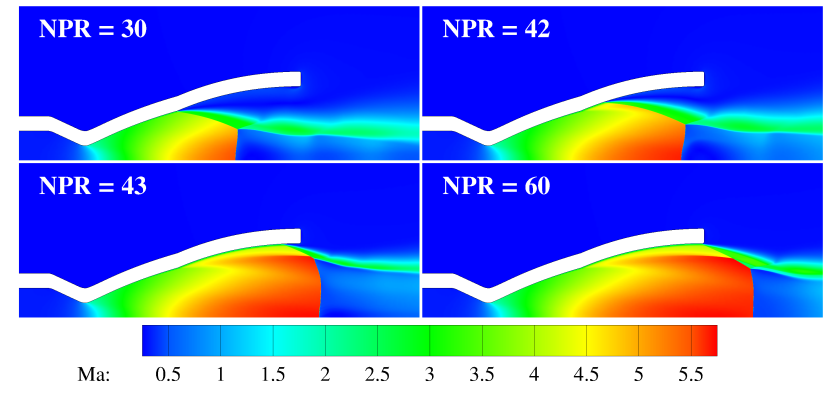

Figure 6. Mach number distribution for different values of NPR.

Figure 7 depicts the experimental data measured by the dynamic pressure transducers of the cold flow test campaign at DLR's test facility P6.2. Each experimental point indicates the nozzle pressure ratio at which the flow attaches to the nozzle extension wall, indicated by a pressure drop at the corresponding sensor position. Thus, the experimental transition NPR was determined to 47.7. In the numerical results, the flow first attaches to the nozzle end at NPR 43. The difference of NPR 4.7 means a deviation from the experimental data of approx. $10 \%$.

Figure 8 illustrates the experimental and numerical wall pressure distribution for the dual bell nozzle in sea-level mode at NPR 30, in altitude mode at NPR 48 and shortly before and after numerical transition at NPR 42 and 43. As long as the flow is attached to the nozzle wall the numerical results show good agreement

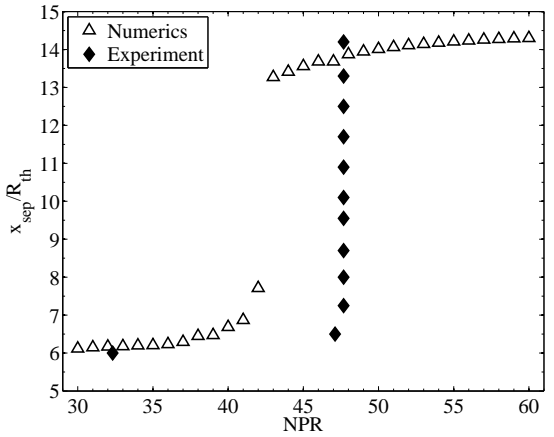

Figure 7. Comparison of numerical and experimental transition NPR.

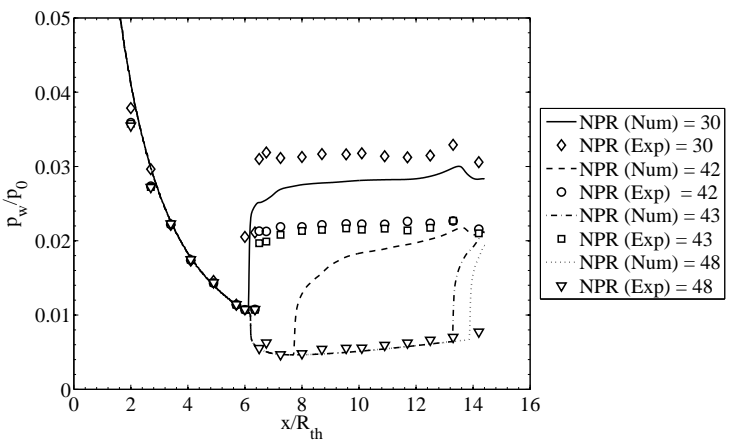

Figure 8. Wall pressure distribution for different values of NPR.

with the experimental data. A strong pressure drop in the throat region and the TIC base nozzle can be observed. When the flow reaches the dual bell contour inflection at $x / R_{t h}=6.2$, an additional expansion occurs and the pressure drops again. A short region of negative wall pressure gradient follows the flow 
expansion at the contour inflection. During the transition process, this negative wall pressure gradient leads to sneak transition whereby the flow separation position moves slowly into the dual bell nozzle extension as shown in Figure 5. When the flow separation position passes this region, comparable flow separation behavior like in conventional nozzles occurs. The flow separates asymmetrically, which induces undesirable strong side loads on the nozzle. Hence, the region of negative wall pressure gradient should be small to avoid this side loads. Downstream of the region with negative wall pressure gradient, a clear positive pressure gradient along the nozzle's pp extension can be observed. The wall pressure increases steadily in the flow direction. For the dual bell nozzle in sea-level mode, the flow separates in the vicinity of the contour inflection. An oblique separation shock leaves the separation position, hits the Mach disk and is reflected in radial direction. Between the core flow downstream of the Mach disk and the atmosphere a shear layer develops. The atmospheric air is sucked inside the nozzle and forms recirculating flow between the shear layer and the inner wall of the nozzle as shown in Figure 1. In this region, the experimental wall pressure illustrated in Figure 8 is almost constant at $90 \%$ of the atmospheric pressure level. In the numerical data, the wall pressure in the recirculation zone is underdetermined by the Spalart-Allmaras turbulence model and decreases in upstream direction. A clear increase of wall pressure at the flow separation position can be observed.

Schlieren imaging was conducted during the cold flow test campaign, to investigate the position and structure of the shock pattern in the nozzle exhaust flow. Figure 9 illustrates the density gradient of the numerically investigated nozzle flow for the dual bell nozzle in altitude mode. Experimental data points read out of the Schlieren images at NPR 49 are plotted in the numerical Schlieren image at the same NPR. Good agreement can be observed between experimental and numerical data for the positions and angles of the oblique shock and the Mach disc. In the vicinity of the triple point the numerical Mach disc is bent in upstream direction. Thus, the triple point and the reflected shock are shifted in radial and upstream direction. However, the numerically obtained shock pattern shows good agreement with the experimental data.

Numerical simulations with different approaches for solving

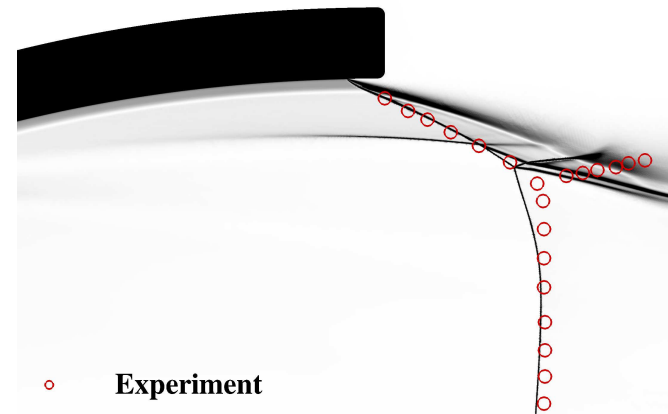

Figure 9. Comparison of numerical and experimental shock pattern position at NPR 49. the RANS equation's closure problem were conducted, to investigate the impact of the applied turbulence model. Because of the interest in the flow behavior near the nozzle wall, the Wilcox $\mathrm{k}-\omega^{13}$ and the Menter $\mathrm{SST}^{14}$ two-equation turbulence models were compared with the Spalart-Allmaras ${ }^{8}$ one-equation turbulence model and a Reynolds-Stress-Model following the approach of Launder et al. ${ }^{15}$ As illustrated in Figure 10 the two-equation turbulence models yield a comparable tran-

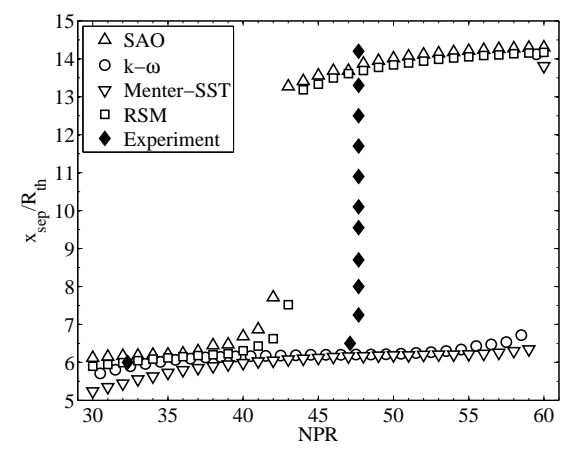

Figure 10. Steady state transition behavior of the investigated dual bell nozzle applying different turbulence models.

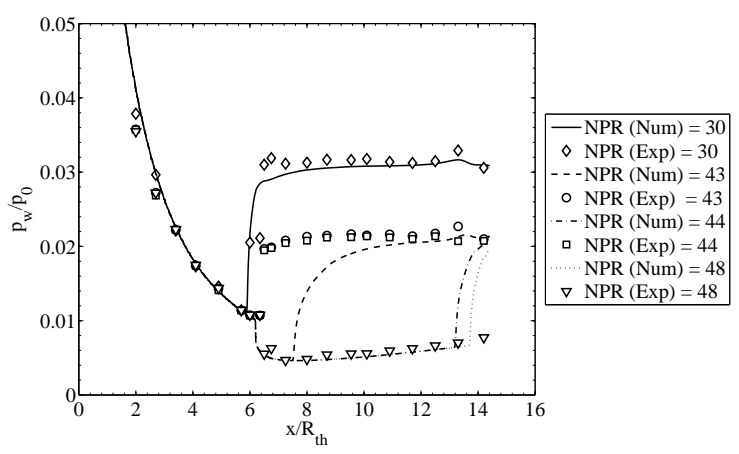

Figure 11. Wall pressure distribution for different values of NPR applying the Reynolds-Stress-Model.

sition behavior. The Wilcox $\mathrm{k}-\omega$ and the Menter SST turbulence models display too sensitive behavior to flow separation. Between NPR 30 and 59 the dual bell nozzle flow separates in the vicinity of the contour inflection and the nozzle stays in sea-level mode for too wide a range of nozzle pressure ratios. Transition- 
NPR for the two-equation turbulence models was determined to be NPR 60, which is a deviation of $26 \%$ from the experimental data. The Reynolds-Stress-Model yields a similar flow separation behavior to the Spalart-Allmaras turbulence model. It can be observed that for the RSM case, the dual bell nozzle reaches altitude mode at a nozzle pressure ratio of 44, which corresponds to a deviation of approx. $8 \%$ from the experimental data. Figure 11 illustrates the comparison of the numerical wall pressure distribution gained by the Reynolds-Stress-Model and the experimental data. In the recirculation zone of the dual bell nozzle flow in sea-level mode, the agreement is better than that obtained by the Spalart-Allmaras turbulence model shown in Figure 8. However, due to the main interest in separation behavior, higher stability, and lower computational cost, the Spalart-Allmaras turbulence model was chosen for further investigation.

\section{Transient Transition Behavior}

The previous steady state RANS simulations yield no information about transition duration and hysteresis behavior of the dual bell nozzle with pp extension. To investigate these significant parts of dual bell nozzle behavior, URANS simulations were conducted. The initial condition for the transient simulations was a fully converged steady state result at a low NPR. Starting from this solution, the feeding pressure of the

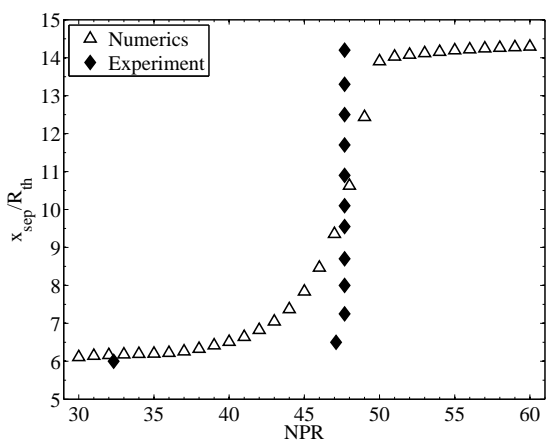

Figure 12. Comparison of numerical and experimental transition NPR for transient simulation.

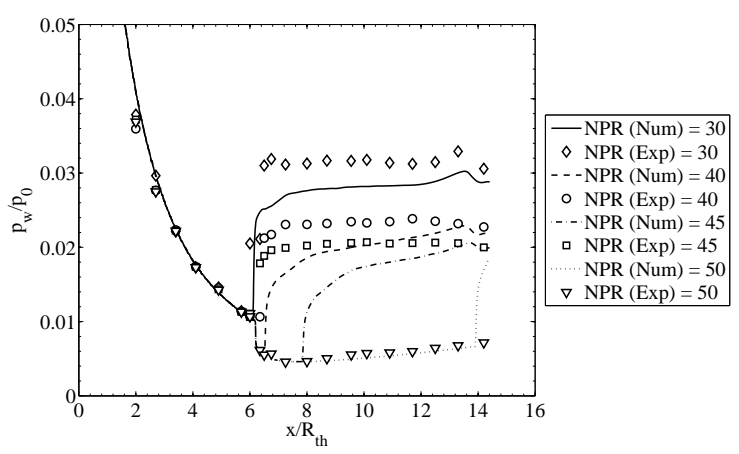

Figure 13. Wall pressure distribution for different values of NPR for transient simulation.

reservoir pressure boundary condition was increased linearly applying a Jameson-type ${ }^{11}$ dual time-stepping scheme. A sensitivity study was conducted to investigate the impact of the physical time step on the dual bell transition behavior. Between $5 \cdot 10^{-7}$ and $1 \cdot 10^{-5} \mathrm{~s}$ almost no influence was observed. Thus, a physical time step of $1 \cdot 10^{-6} \mathrm{~s}$ was applied.

Figure 12 shows the separation curve for a transient simulation applying a feeding pressure ramp of $2.5 \mathrm{bar} / \mathrm{ms}$. This is three orders of magnitude faster applied feeding pressure ramp than in the experiments, because of limited computational power. Below NPR 40 and above NPR 49 a clear sea-level mode and altitude mode can be observed, respectively. Between NPR 40 and 49, no sudden shift of the flow separation position like that obtained with the steady state simulations takes place. A more progressive movement of the flow separation position inside the nozzle extension is observed. To determine the transition NPR of the numerical results and compare it with experimental data, a definition as described in Génin et al. ${ }^{16}$ was made. Thus, the inflection point of the flow separation position evolution curve was defined as transition NPR of the numerical simulations. The comparison of the numerical results and the experimental data shown in Figure 12 yields good agreement. The deviation of the numerically determined and the experimentally measured transition NPR is less than $1 \%$. For both approaches transition takes place between NPR 47 and 48. This transition NPR prediction accuracy was also shown in Génin et al. ${ }^{16}$ for a dual bell nozzle with constant pressure extension. Figure 13 illustrates the wall pressure distribution for the conducted URANS simulations. The comparison with the experimental data for different NPR steps yields the same conclusion as described for the steady state simulations.

To ensure a stable altitude mode against pressure fluctuation in the combustion chamber and the environment, the hysteresis gap between transition to altitude mode and retransition to sea-level mode has to be maximized. Thus, the hysteresis behavior of the current nozzle model with pp extension was investigated. Therefore, a URANS simulation applying down-ramping of the inlet pressure was carried out. As initial condition for the down-ramping process a result at high NPR of the up-ramping simulations was used. 
Starting from this solution the feeding pressure was decreased linearly until sea-level mode was reached. Figure 14 illustrates the flow separation position over NPR for the up- and down-ramping simulations. It

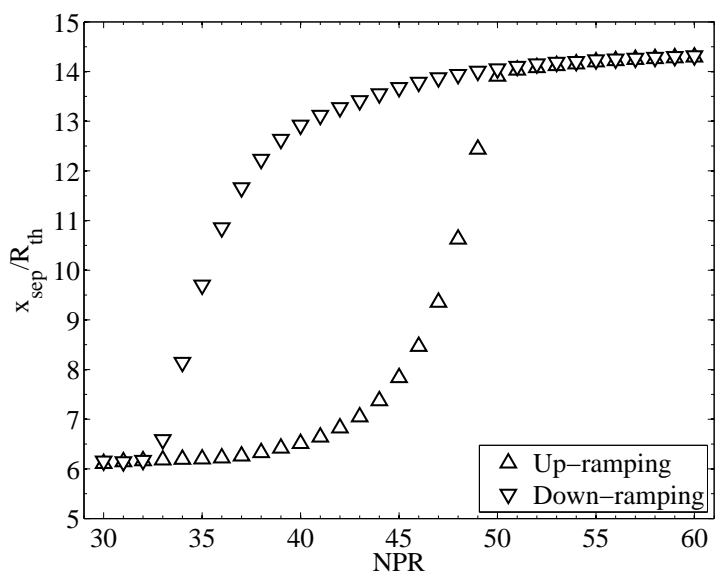

Figure 14. Hysteresis behavior of the dual bell nozzle with pp extension.

can be observed that in low sea-level and in high altitude mode the flow separates exactly at the same positions in the dual bell nozzle. However, during the transition/retransition process, the nozzle behaves differently depending on the direction of the inlet pressure ramp. If the flow separation position evolution curve's inflection point is again defined as transition NPR, the retransition NPR of the investigated dual bell nozzle is determined to be 33.5. Thus, the numerical hysteresis gap:

$$
H=\frac{\mathrm{NPR}_{\mathrm{tr}}-\mathrm{NPR}_{\mathrm{retr}}}{\mathrm{NPR}_{\mathrm{tr}}} \cdot 100
$$

amounts to $30 \%$. Considering the experimental retransition NPR of 29.8 , the numerics underdetermine the hysteresis gap of the investigated dual bell nozzle by approx. $5 \%$ points.

As described before the experimental and numerical feeding pressure gradients applied for the transient flow simulations differed by three orders of magnitude. An influence of the inlet pressure ramping speed of a dual bell nozzle with cp extension was already shown by Wong. ${ }^{17}$ Transient simulations with different

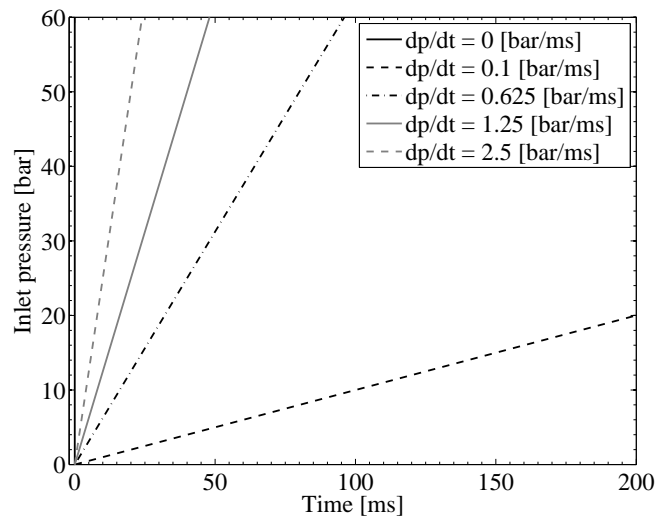

Figure 15. Different feeding pressure gradients for transient investigations.

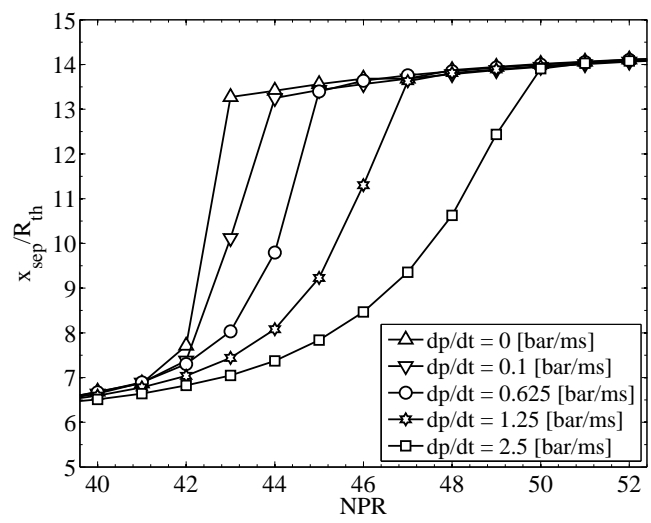

Figure 16. Influence of feeding pressure gradient on transition behavior.

feeding pressure ramps were conducted, to investigate the impact of the feeding pressure gradient on the dual bell transition for nozzle with a positive pressure gradient along the nozzle extension. Figure 15 shows the inlet pressure evolution over time for the steady state case and the four different ramping speeds between 2.5 and $0.1 \mathrm{bar} / \mathrm{ms}$. Figure 16 illustrates the dedicated transition evolution curves. With decreasing feeding 
pressure gradient a shift of the transition evolution curve in the direction of the steady state result can be observed. Thus, the transition turns to a more abrupt process if the feeding pressure ramp is changed in the direction of the experimental value. It can be assumed that using the physical feeding pressure gradient of the experiments would yield the same results as the steady state simulations. This means that applying the experimental inlet pressure ramp yields a quasi-stationary transition behavior of the dual bell nozzle. Figure 17 illustrates the speed of the flow separation position moving along the dual bell nozzle extension.

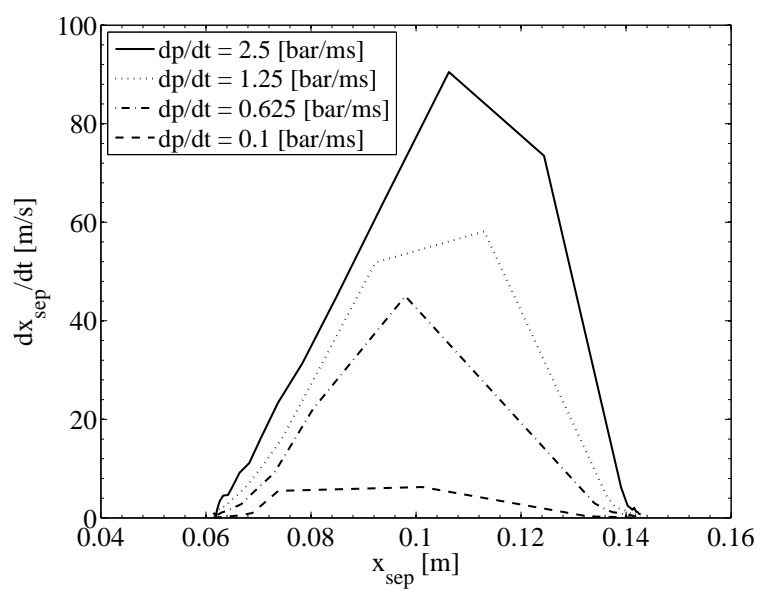

Figure 17. Speed of the flow separation position for different feeding pressure gradients.

The separation position speed increases when the flow separation leaves the contour inflection. For all cases the maximum speed is reached in the middle of the contour inflection. This agrees with the experimental observations. ${ }^{4}$ After reaching the maximum the velocity decreases again until the exit plane of the nozzle is reached. The highest value of separation position speed is approx. $90 \mathrm{~m} / \mathrm{s}$ for a feeding pressure gradient of $2.5 \mathrm{bar} / \mathrm{ms}$, which is in the experimental order of magnitude. With decreasing feeding pressure ramp the velocity of the separation position decreases too. The clear impact of the inlet pressure gradient on the speed of the flow separation position along the dual bell nozzle extension can be observed in Figure 17.

\section{Conclusion}

RANS and URANS simulation have been conducted using DLR's in-house flow solver TAU, to investigate the transition behavior of a dual bell nozzle with pp extension. Validation data for the numerical simulations were obtained by a cold flow test campaign at DLR's test facility P6.2. The comparison of the steady state simulation transition NPR and the experimental value yielded a deviation of approx. $10 \%$. Good agreement was achieved by comparing the shock pattern of the simulations with experimental data obtained by Schlieren imaging. For the nozzle in sea-level mode, the wall pressure along the recirculating flow area was underestimated by the numerical simulations. Better results for the wall pressure distribution were achieved by applying the RSM turbulence model.

URANS simulation were carried out, to investigate the transient dual bell transition phenomena. Applying a time step of $1 \cdot 10^{-6} \mathrm{~s}$ and a feeding pressure gradient of $2.5 \mathrm{bar} / \mathrm{ms}$ yielded a deviation of less than $1 \%$ from the experimental transition NPR. This accuracy was already shown in Génin et al. ${ }^{16}$ for a dual bell nozzle with cp extension. A down-ramping of the feeding pressure was conducted to investigate the hysteresis behavior of the dual bell nozzle. The retransition NPR was determined with an accuracy of approx. $12 \%$, the hysteresis gap was underdetermined by approx. $5 \%$ points. To investigate the impact of the feeding pressure gradient on the dual bell transition behavior, simulations with different feeding pressure ramps were conducted. With decreasing feeding pressure gradient a shift of the transition NPR in the direction of the steady state simulations was observed. Thus, applying the experimental inlet pressure ramp the dual bell transition is identified as a quasi-stationary process. The velocity of the flow separation position during transition from sea-level to altitude mode reached its maximum in the middle of the dual bell nozzle extension and is in good agreement with the experimental data. Decreasing the feeding pressure gradient yielded slower flow separation position movement along the dual bell nozzle extension wall. 
The numerical model developed in the present work is able to predict the transition behavior of a cold flow dual bell nozzle with sufficient accuracy. The reliable prediction of the transition NPR, the retransition NPR, and the hysteresis gap can reduce the cost for experiments and is the basis for future work. Full 3D investigations conducted by the authors yielded almost no influence of $3 \mathrm{D}$ effects on the dual bell transition behavior. In the future, the authors will extend the developed numerical model by a chemical reaction mechanism, to study the dual bell transition behavior under hot flow conditions. ${ }^{18}$

\section{Acknowledgments}

Financial support has been provided by the German Research Foundation (Deutsche Forschungsgemeinschaft) in the framework of the Sonderforschungsbereich Transregio 40. Special thanks goes to the colleagues from DLR Goettingen (TP B5) for their assistance. The authors would like to thank the members of the DLR internal collaboration ProTAU for supporting the numerical studies.

\section{References}

${ }^{1}$ Foster, C. R. and Cowles, F. B., "Experimental Study of Gas-Flow Separation in Overexpanded Exhaust Nozzles for Rocket Motors," Technical report, Jet Propulsion Laboratory, 1949.

${ }^{2}$ Horn, M. and Fisher, S., "Dual-Bell Altitude Compensating Nozzles," Tech. Rep. CR-194719, NASA, 1994.

${ }^{3}$ Schneider, D., Génin, C., Stark, R., and Fromm, C. M., "4th Space Propulsion Conference," Ariane 5 Performance Optimization Using Dual Bell Nozzle Extension, Cologne Germany, 2014.

${ }^{4}$ Génin, C. and Stark, R., "Experimental Study on Flow Transition in Dual Bell Nozzles," Journal of Propulsion and Power, Vol. 26, No. 3, May 2010, pp. 497-502.

${ }^{5}$ Génin, C., Stark, R., and Schneider, D., "49th AIAA/ASME/SAE/ASEE Joint Propulsion Conference \& Exhibit," Transitional Behavior of Dual Bell Nozzles: Contour Optimization, Atlanta, USA, 2013.

${ }^{6}$ Schwamborn, D., Gerhold, T., and Heinrich, R., "European Conference on Computational Fluid Dynamics," The DLR TAU-Code: Recent Applications in Research and Industry, Delft, Netherlands, 2006.

${ }^{7}$ Mack, A. and Hannemann, V., "32nd AIAA Fluid Dynamics Conference and Exhibit," Validation of the Unstructured DLR-TAU-Code for Hypersonic Flows, St. Louis, USA, June 2002.

${ }^{8}$ Spalart, P. R. and Allmaras, S. R., "30th Aerospace Sciences Meeting \& Exhibit," A One-Equation Turbulence Model for Aerodynamic Flows, AIAA, Reno, January 6-9 1992.

${ }^{9}$ Stark, R. and Hagemann, G., "2nd European Conference for Aerospace Sciences (EUCASS)," Current Status of Numerical Flow Prediction for Separated Nozzle Flows, Brussels, Belgium, July 2007.

${ }^{10}$ Wada, Y. and Liou, M.-S., "32nd Aerospace Sciences Meeting \& Exhibit," A Flux Splitting Scheme with High-Resolution and Robustness for Discontinuities, Reno, USA, January 1994.

${ }^{11}$ Jameson, A., "10th Computational Fluid Dynamics Conference," Time Dependent Calculations Using Multigrid, with Applications to Unsteady Flows Past Airfoils and Wings, Honolulu, USA, June 1991.

${ }^{12}$ Whitfield, D. L., "Three Dimensional Unsteady Euler Equations Solution Using Flux Vector Splitting," Tech. Rep. CR-173254, NASA, 1983.

${ }^{13}$ Wilcox, D. C., Turbulence Modeling for CFD, DCW Industries, 2nd ed., 1994.

${ }^{14}$ Menter, F. R., "Two-Equation Eddy-Viscosity Turbulence Models for Engineering Applications," AIAA Journal, Vol. 32, No. 8, August 1994, pp. 1598-1605.

${ }^{15}$ Launder, B. E., Reece, G. J., and Rodi, W., "Progress in the Development of a Reynolds-Stress Turbulent Closure," Journal of Fluid Mechanics, Vol. 68, 1975, pp. 537-566.

${ }^{16}$ Génin, C., Stark, R., Karl, S., and Schneider, D., "48th AIAA/ASME/SAE/ASEE Joint Propulsion Conference \& Exhibit," Numerical Investigation of Dual Bell Nozzle Flow Field, Atlanta, USA, July 2012

${ }^{17}$ Wong, H. and Schwane, R., "Fourth Symposium on Aerothermodynamics for Space Vehicles," Numerical Investigation of Transition in Flow Separation in a Dual-Bell Nozzle, Capua, Italy, October 2001.

${ }^{18}$ Génin, C., Schneider, D., Tomita, T., and Takahashi, H., "51st AIAA/SAE/ASEE Joint Propulsion Conference \& Exhibit," LOX/CH4 Hot Firing Dual Bell Nozzle Testing: Part I -Transitional Behavior-, Orlando, USA, 2015. 